\title{
SOSTENIBILIDAD EN LA GESTIÓN DEL DISEÑO DE PRODUCTO: EL VALOR DEL DISEÑO EN LA EMPRESA.'
}

Sustainability in Product Design Management: The value of design in the company

Fecha de recepción: 13 de septiembre de 2019 Fecha de aceptación: 27 de diciembre de 2019 


\section{RESUMEN}

Este documento, expone una visión más amplia de la disciplina del diseño dentro de las empresas. Usualmente, las empresas encasillan el trabajo del diseñador, en áreas como comunicación corporativa, identidad e imagen de marca, diseño de productos y diseño de espacios. Sin embargo, aqui dejaremos ver la importancia de la visión del diseño, desde una perspectiva incluyente, holística y multidisciplinaria dentro de las empresas. Explicando así la relación del diseño, en la toma de decisiones de los bienes o servicios, el desarrollo de los productos y el impacto que esto genera sobre la solución de problemas, desde un punto de vista crítico y responsable. Finalmente, podremos apreciar la intervención y conocimiento en la gestión del diseño, esto independientemente del tamaño de la empresa; la responsabilidad del diseño y su actividad mediadora definirán, su interacción con los individuos y su entorno a través de la sostenibilidad.

Palabras clave: Diseño, Empresa, Producto, Responsabilidad, Sostenibilidad

\section{ABSTRACT}

This document exposes a wider vision in the discipline of design within the business environment. Often, businesses limit the work of designers to areas such as corporate communication, brand image or identity and product or space design. However, here we aim to show design's relevance and importance from a more inclusive, holistic and multidisciplinary perspective within the business environment. Explaining the relationship between design and the decision-making process for goods and services, product design and the impact this may have in problem solving processes from a responsible and critical point of view. Finally, we may appreciate the intervention and knowledge granted by design management, disregarding the company size; design responsibility and its mediator capabilities will ultimately define its interaction with individuals and the environment through sustainability

Keywords: Design, Business, Product, Responsability, Sustainability

Clasificación JEL: M10, M14, M31. 


\section{Introducción}

$\mathrm{E}$ 1 Diseño, ha sido una parte indispensable dentro de las empresas; más de lo que pudiéramos llegar a reconocer. Muchos países han logrado dar un mayor valor dentro de sus instituciones, haciéndolo parte de su identidad. Ejemplo claro de ello están los italianos en el diseño de mobiliario, los franceses en el diseño de modas, los alemanes contribuyendo con sus diseños automotrices y los japoneses y españoles reconocidos por sus diseños de marca, esto solo por mencionar algunos.

El diseño está en todas partes, en todo lo que percibimos de forma natural como; las texturas de los árboles, sus hojas, una nube, una roca, un coral, etc., también, está el diseño que es creado por el hombre, a través de objetos o servicios, con características o particularidades que definen una identidad y aplicación utilitaria.

Es por ello por lo que al diseño se le define como, la capacidad humana para crear en base a satisfacer y mejorar las necesidades del hombre para el hombre, en todos los ámbitos en los que se encuentra inmerso. Dentro de algunas reglas básicas del diseño, encontraremos que este deberá tener una utilidad, en su función, y su forma, así como, el material con el que se elabora, etc.

\section{El diseño dentro de las empresas}

El diseño cuenta con un sin fin de áreas o especialidades como: diseño industrial, gráfico, de interiores, digital, de producto, de moda, de autos, de imagen, de comunicación, de multimedios, solo por mencionar algunos, y dentro de cada una de estas disciplinas del diseño, encontraremos una enorme variedad de especialidades como: la imagen, la marca, los medios los procesos, los costos, la planeación, etc., en donde existe una relación intrínseca, entre todas y cada una de las funciones y de sus áreas de especialidad. Es justamente en una empresa, en donde mejor se muestra, la participación del diseño, solo por dar un ejemplo; La identidad corporativa se manifiesta en tres ámbitos:

1. Productos y servicios (lo que fabrica),

2. Entornos (donde fabrica o vende), y

3. Comunicaciones (es decir como lo hace).

La imagen corporativa o imagen institucional, nos permite poder identificar una empresa de otra, conocer sus atributos, mismos que tendrán toda la intención de mostrar a su entorno y sociedad, así como sus características principales. Conjuntamente, visualiza el ser reconocida y como la gente le describe y le recuerda; de este modo buscará ser un icono, el cual remita un conjunto de significados visuales. Es decir, al momento de verle; este generará un discurso de todo lo que oferta y representa.

La percepción, que la gente guarda en la memoria, es otro importante referente que deberá considerar la empresa. Esto puede construirse a través de la propia experiencia, usualmente estos son aspectos que se utilizan en una investigación de mercado. El grado de participación del diseño, interviene desde el desarrollo del cumulo de conocimientos, atributos y actitudes que proyecte un objeto-imagen para ser reconocido. 
Capriotti (2013) menciona que existen diferentes expresiones para referir lo mismo; imagen global, imagen integral, imagen de marca de la empresa, etc. Aquí se menciona que, el problema no radica en el número de expresiones, sino como estas se aplican. Incluso, indica que el problema no radica en la definición etimológica, sino en su adecuada aplicación. De este modo, solo se centra en el aspecto de la imagen, como una representación de un objeto real que puede ser, una Imagen-Ficción, una Imagen-Icono o una Imagen-Actitud. Cada una de ellas, es una representación mental, siendo la idea que construimos, en donde se otorgan algunos atributos con los que se busca definir y diferenciarse, para generar un significado específico en cada receptor (Capriotti, 2013).

El diseño de la marca es uno de los puntos importantes, pero cuidado, no debemos confundir esto con el logotipo, el nombre, o las letras (tipografía) con el que se identifica o bien, con lo que la marca significa. La marca se entiende, como el beneficio que percibe para, el cliente, mismo que se identificará a través de un nombre, un producto, una empresa, servicio o negocio. Es por ello que, buscará significar algo, mostrar cuál es su propósito, permitiendo establecer o conocer entre sus clientes o consumidores, las diferencias con respecto de su competencia. Llegar a lograr esto, es una cuestión compleja, algunos clientes caen en la trampa de pensar que el diseño de una marca está relacionado a la representación icónica de su nombre o logo. La marca es la que determinará al final del día, como los individuos perciben al producto servicio, empresa o negocio.

"Es cualquier nombre, termino, signo, símbolo, diseño o cualquier combinación de estos elementos, cuyo propósito consiste en identificar los bienes o servicios de uno o varios vendedores y en diferenciarlos del resto de los compradores" (Kotler y Amstrong, 2003, p. 298).

Es importante mencionar a Norberto Chaves, quien en su libro La imagen corporativa comenta que la identidad son una serie de atributos, intrínsecos de la institución además conformados por un conjunto de signos identificadores; asimismo, opina que es común el confundir los términos entre la identidad y la imagen. Siendo que la identidad, constituye un sistema de mensajes complejos en los cuales identifica tres grupos: los signos; el nombre los logotipos, la versión grafica del nombre; que es la marca y los imagotipos; es la representación gráfica, en donde el icono y el nombre de la marca conforman una unidad visual (Chaves, 2003).

Consecuentemente, en el diseño de marca encontraremos la Identidad de la Marca; que es todo aquello que la conforma; sus cualidades tangibles o intangibles dándolo un valor único entre otras y la Imagen de la marca, esta se obtiene a través de la activación de la marca en los diferentes puntos de contacto, es decir, es la construcción de esta.

A diferencia de Chaves, Capriotti (2013) menciona cinco diferentes niveles de Imagen dentro del ambiente empresarial: Imagen de producto Genérico, Imagen de marca de producto o servicio, Imagen corporativa o de marca corporativa, Imagen de sector empresarial e imagen de país. Estableciendo que la imagen corporativa sería el conjunto de rasgos o atributos que se le otorgan a la empresa, los diferentes públicos, al procesar la información relativa a la misma.

De este modo, la participación del diseño es fundamental, ya que se vuelve un factor decisivo en el proceso de la adquisición de compra o reconocimiento del producto o servicio. Sin embargo, en ocasiones la 
marca se encuentra apartada de las características o percepciones y atributos del producto; usualmente se le atribuyen a la empresa, el servicio o al negocio, dichas cualidades.

El producto, incorpora elementos que trascienden e impactan en muchas áreas, algunas de ellas, es factible que ni los mismos diseñadores estén considerando, como puede ser su grado de innovación, la calidad de la solución del diseño, los costos etc. Este proceso deberá considerar una intervención multidisciplinaria desde antes (el diseño del producto), durante (el proceso del desarrollo del producto) y después (la gestión del producto). La etapa previa al diseño el antes; interviene la planeación de un producto, su conceptualización la competencia, el posible grupo meta, materiales, almacenaje, línea de producción ya sea un nuevo diseño o bien, lo que se conoce como un rediseño. La etapa del durante el desarrollo; interviene un gran equipo asignado a la toma de decisiones ya que cualquier consideración puede impactar en diferentes áreas dentro de la empresa como: costos, los procesos de producción, el empaque y embalajes, en los materiales, solo por mencionar algunos. Así, por mínimo que pudieran llegar a considerarse algún cambio en el producto, este deberá pasar por una serie de aprobaciones o desaprobaciones. El diseñador debe ser participe y estar en contacto con todas las áreas y fases para estar consciente de las propuesta e impactos de cualquier alteración en el producto. Cabe mencionar que esta, es quizá la parte en donde las empresas tienen mejor identificada, la participación del diseño.

El modelo de producto integral se define como la suma de elementos básicos esperados, adicionales y potenciales. En donde los elementos básicos son aquellos elementos mínimos imprescindibles con los que debe contar el producto. Los elementos esperados; son los que el consumidor espera cuente el producto con ellos. Los elementos adicionales; serán los elementos que marcarán la diferencia entre otros de su misma línea. Elementos potenciales; usualmente son intangibles, pero cuentan con un valor (Press y Cooper, 2009).

La comunicación dentro de una empresa puede ser en dos sentidos; de manera interna y de manera externa. En la comunicación interna incluye básicamente a todos los integrantes de la misma empresa, ya sea para dar algún anuncio, informe, indicación de forma individual o grupal y esta puede ser de forma ascendente o descendente; depende claro de la estructura jerárquica que presente la empresa o institución. Algunos de los medios que utiliza van desde; cartas personales, algún periódico semanal o mensual, diseño de material para reuniones informativas, presentaciones, folletos, trípticos, dípticos, volantes, infografías, señalética, señalizaciones, manuales de uso, cursos, anuncios impresos o digitales, solo por mencionar algunos.

En la comunicación externa de una empresa los mensajes esta dirigidos a crear o mantener la relación o información con los diferentes grupos meta, además de proyectar una imagen e identidad de la empresa, promover algún evento producto o servicio; el trabajo del diseño se centra en el diseño de páginas web, comunicación con la prensa, o cualquier otro tipo de medio impreso o digital, televisión, radio, correos electrónicos, invitaciones etc.

Los medios de comunicación son de vital importancia para una empresa, y existe una enorme variedad en los que puede apoyarse. Mantener una constante línea de diseño e información, dará identidad e imagen, generando confianza.

Las empresas destinan una parte importante de su tiempo y sus recursos a la obtención, el proceso, la aplicación y la proyección de información. Es por ello por lo que la comunicación juega un papel preponderante para las empresas ya que puede llegar a convertirse en su principal patrimonio. Esta deberá ser clara, precisa y que se adapte a la formación y perfil de quien va dirigida (Urrutia, 2000). 


\section{La función del diseño}

El diseño en una empresa no es una moda es parte integral de muchas de las áreas. En este documento apenas hemos esbozado una pequeña revisión la relación intrínseca entre las áreas del diseño y la empresa. El diseño no solo es dibujar bonito.

La disciplina del Diseño busca solucionar problemas; si consideramos que la mayoría de las ocasiones los problemas son multifactoriales, es importante considerar que para ello se requiere de un proceso de investigación un diseño metodológico el cual incluye una investigación un análisis, pruebas previas (de campo o técnicas), ajustes, registros de documentación; hasta llegar a una propuesta final, sea en cualquiera de las intervenciones que se plantearon con anterioridad. Aquí, los problemas pueden ser de comunicación interna ó externa, de diseño o rediseño del producto, de diseños de empaque ó de embalaje, del posicionamiento del producto o de la marca etc.

El diseño también viste a las empresas; contar con una identidad y una imagen, como ya anteriormente se mencionó, es muy importante, esto es gracias a la importancia que se establece en el producto, en el envase que lo contiene y al mensaje que se estructura cuando se percibe una línea congruente de información y trabajo refleja una identidad, la cual tiene un valor agregado que es un reflejo de la empresa.

Hoy en día nos tomamos el tiempo para revisar y comparar los productos o servicios que se ofertan sobre todo si existe una demanda de productos similares. Es importante reflexionar en cómo percibe el consumidor los atributos del producto o servicio. La forma de venta cada vez es más compleja y cambia rápidamente, por ello conocer la experiencia de los usuarios y los diversos dispositivos por los que se envía dicha información, es cada vez más importante para las empresas y sus diseñadores.

Las empresas perciben una creciente demanda, mayor rapidez en el desarrollo de productos, ciclos de diseño rotación de inventarios y respuesta competitiva, con todo lo que ello conlleva para quienes trabajan ahí. El diseño dentro de una empresa genera identidad, imagen, explica, comunica.

La gestión del Producto es otra de las importantes tareas del diseño dentro de una empresa: Los aspectos que intervienen son la planificación, la previsión, el trabajo de diseño, lo relacionado con la producción, todo lo concerniente con el empaque y embalaje, así como la logística de la distribución, los costos, los estudios de mercado y desde luego la promoción y publicidad del producto o de la marca. Estas son las áreas en las que debe intervenir el diseñador o por lo menos estar presente dentro de la toma de las decisiones ya que de esta forma estará consiente de que cualquier cambio que se solicite tendrá un impacto en cada una de estas áreas. Como se puede apreciar en la figura 1, se muestran los pasos dentro de la gestión del producto, no hay una secuencia específica, y se puede trabajar en conjunto con varias a la vez. 


\section{Figura 1. Pasos en la Gestión del Producto}

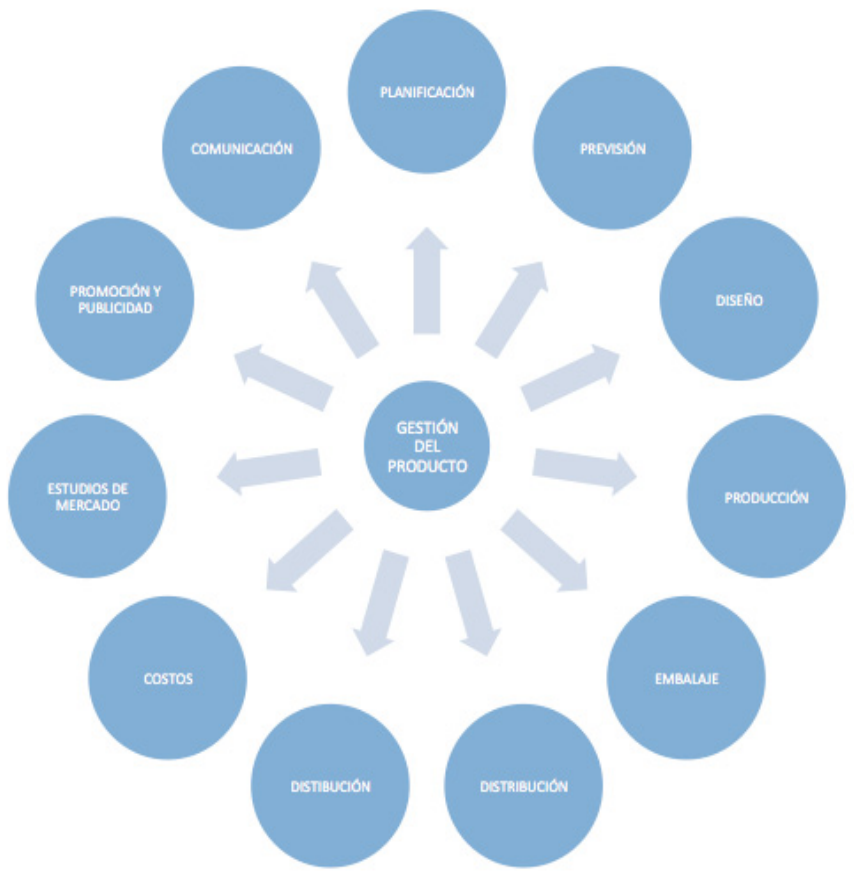

Fuente: Elaboración propia.

La administración del producto es una función muy importante de la actividad empresarial que implica; la definición de las características y beneficios de los productos o servicios en los procesos de desarrollo y fabricación. En el diseño del producto incluye una actividad multidisciplinaria, los pasos que se incluyen son: conocer la historia y la morfología del producto, la estructura, el funcionamiento, la tecnología, el área comparativa o de competencia, la racionalidad del producto y la parte económica. A continuación, en la figura 2, los pasos anteriormente mencionados. Cabe destacar la importancia de la participación del diseñador, en cada una de las áreas, así como trabajar en de forma multidisciplinaria. 


\section{Figura 2. Diseño del producto}

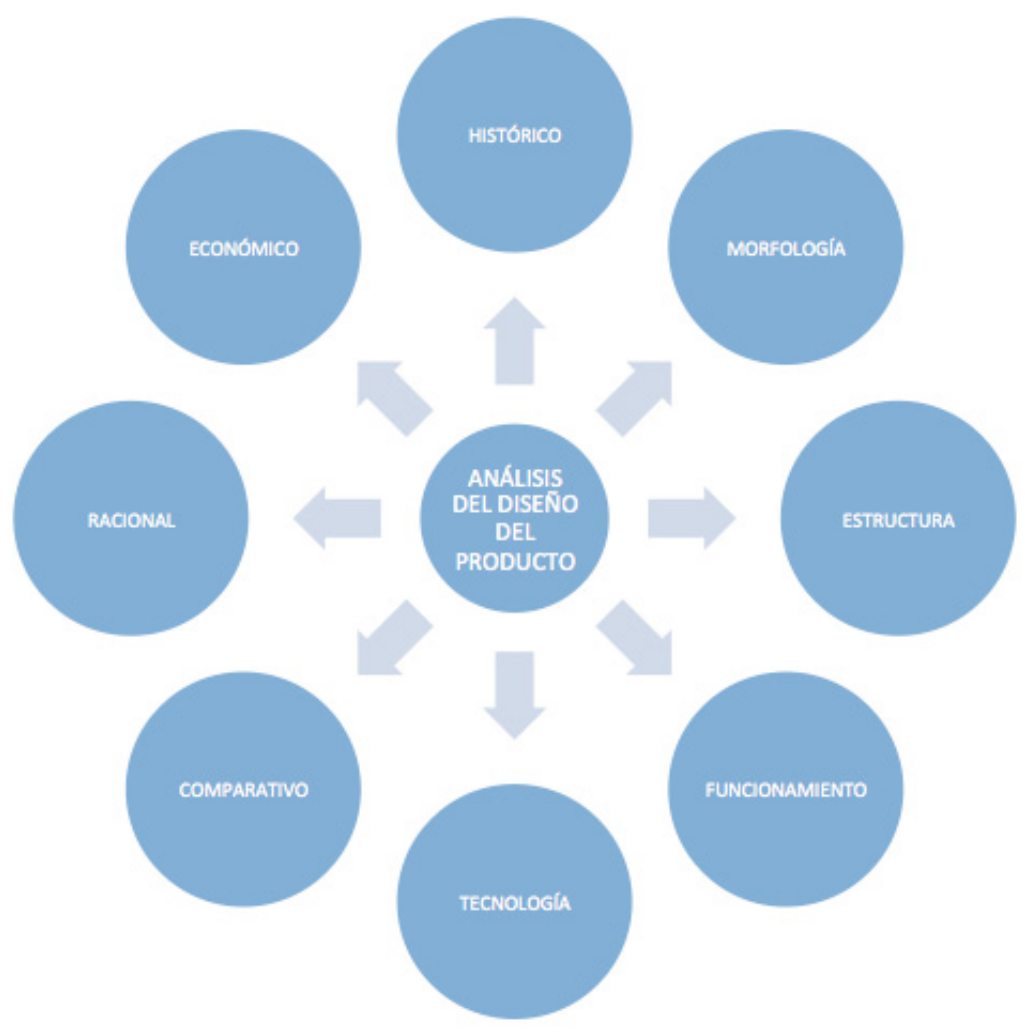

Fuente: Elaboración propia.

El proceso del desarrollo del producto es otra basta área en donde la participación del diseñador es fundamental. Ocupa una gran parte de la empresa algunas de ellas establecen departamentos para las siguientes áreas: gestión de la información precios de transferencias, presupuestos, conocimiento del producto, diversos usos, sus aplicaciones, procesos de fabricación y estudio de la competencia. Lo recomendable es trabajar de forma conjunta ya que igual que en las áreas anteriores la comunicación es fundamental y cualquier decisión siempre tiene un impacto en alguna de las otras áreas en la figura 3. 
Figura 3. Gestión de la información

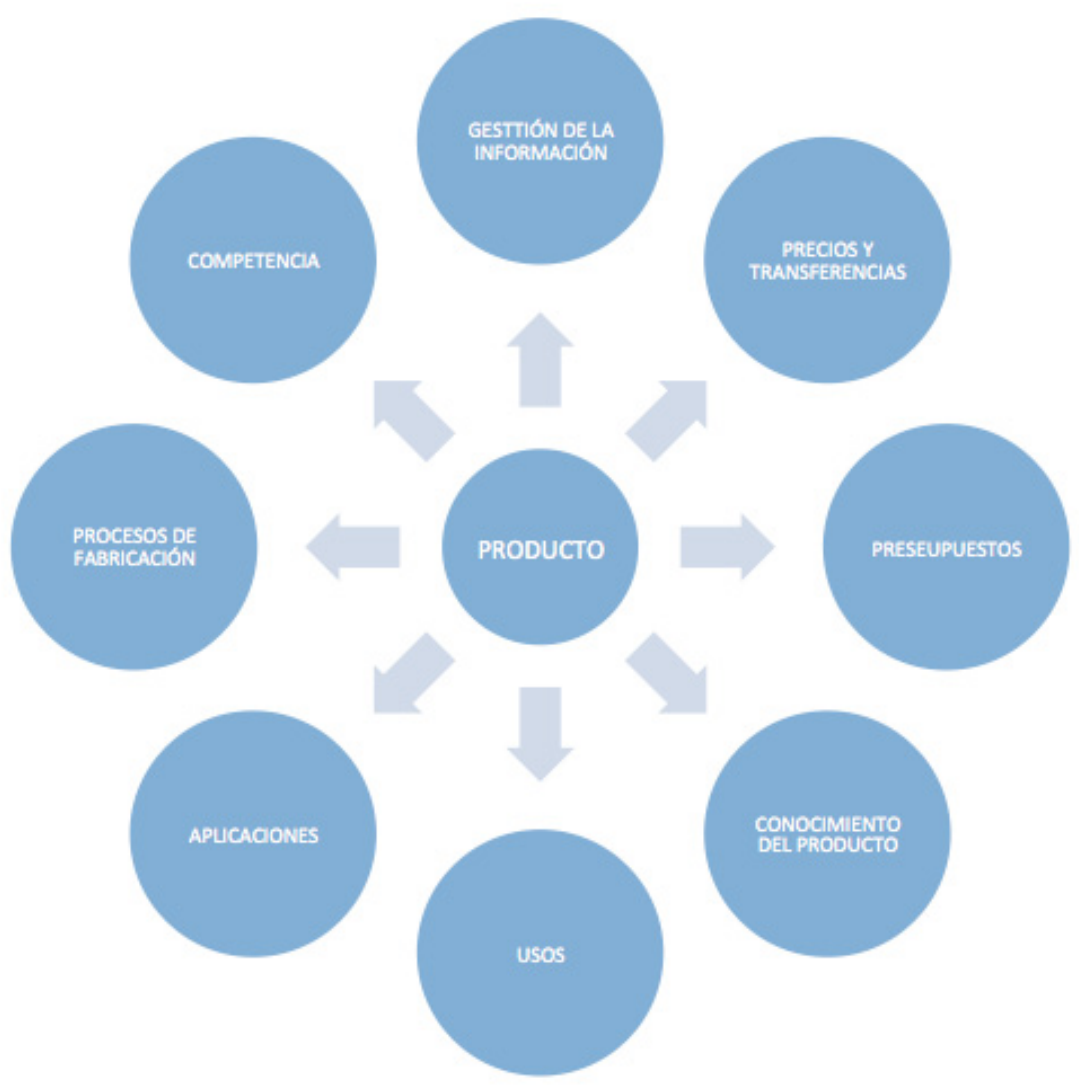

Fuente: Elaboración propia.

Estos ciclos se van desarrollando en lo individual, dependiendo el tamaño de cada empresa y lo óptimo es que la comunicación fluya en forma ascendente y descendente. Como podemos observar, varias de estas áreas se interconectan y algunos son procesos dependientes o subsecuentes de otros, de ahí la importancia de la comunicación.

Lo anteriormente expuesto son todas las áreas en las que el diseño está incluido, a continuación, revisaremos la función del diseñador, así como la responsabilidad de este.

\section{La responsabilidad del diseño como acción de cambio}

Debemos darnos a la tarea de reflexionar sobre, como hemos entendido el diseño, el proceso de persuasión y el diseño de la publicidad, ya que estos han otorgado mayor valor a generar necesidades de compra; más que al hecho de generar soluciones a necesidades y problemáticas sociales. Si bien se ha discutido que, mucho del desarrollo del diseño busca el confort y mejora del individuo, no debiera 
perderse de vista la responsabilidad, que el desarrollo de cada objeto, servicio o bien que se diseña, tendrá un impacto en el entorno.

No se trata de algo nuevo, se trata de ser mayormente responsables con lo que se diseña. El diseñador, debe incentivar una conciencia crítica, ser más exigente con lo que desarrolla, así como el uso o diseño de los procesos y el impacto que esto genera en el entorno. Un diseño responsable deberá pasar por todas las etapas anteriormente comentadas.

La responsabilidad social, es un trabajo de todos; supone un cumplimiento de principios que se deben desempeñar más allá de las responsabilidades legales, mismas que deben entenderse como un deber personal y por consiguiente institucional. Las instancias gubernamentales, educativas, empresariales y la sociedad en general deberán considerar y atender dichas responsabilidades por cuestiones de ética. Una empresa, incluye una combinación de aspectos legales, éticos, morales y ambientales; siendo una decisión voluntaria, no impuesta. Esta, abarca aspectos internos y externos, los primeros se orientan a los colaboradores o el equipo de trabajo y los segundos a los clientes, proveedores, familias de los trabajadores y entorno social; asimismo, deberá considerar desde misión y visión, el impacto que genera en todos sus procesos de producción al medio ambiente.

\section{Un diseño respetuoso y responsable}

Brezet y Hemel (1997) proponen una clasificación de posibilidades estratégicas para la mejora medioambiental conocida como "Rueda de las estrategias" la cual está dividida en ocho pasos; cada una con algunas subdivisiones, esta propuesta apoya al diseñador a reflexionar, en cada uno de los procesos en los cuales interviene su diseño, mismos que van considerando los problemas medioambientales de sus productos y que en ocasiones se pasan por alto. Con la intención de establecer nuevos conceptos, o bien, optimizar la funcionalidad del producto, o incluso diseñar procesos innovadores, que planten mejoras ambientales, en las diferentes etapas del ciclo de vida del producto o servicio (Ver figura 4). 
Figura 4. Rueda de Estrategias de Eco-Diseño

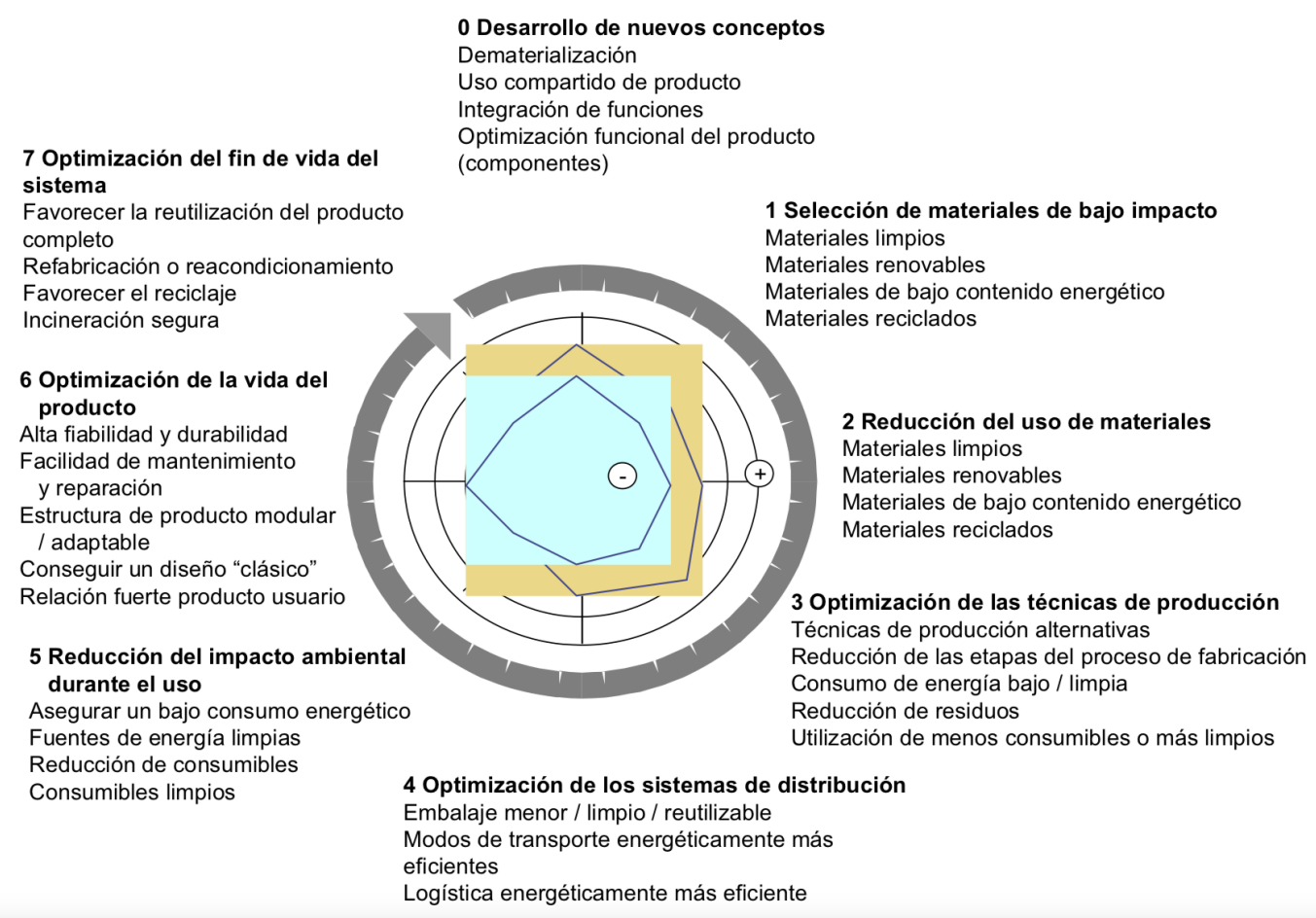

Fuente: Brezet y Hemel (1997).

Como número uno, presenta; la selección de los materiales de bajo impacto. En esta sección, se recomienda el uso de materiales limpios, renovables, materiales de bajo contenido energético, así como materiales reciclados, tales como: PCBs, plomo, cadmio, mercurio, es preferible optar por materiales orgánicos.

Como número dos, reducción del uso de materiales. Aquí, se considerará el uso de materiales limpios, además de reducir el peso y el volumen, ya que cuando se reduce el peso, se supone menos cantidad de material y por lo consecuente menos residuos y en el caso del volumen, se logra la reducción del impacto durante el almacenaje y transporte.

Como número tres, tenemos la optimización de las técnicas de producción. Es necesario elegir tecnologías de producción más limpias. Al momento de reducir la fabricación, se minimiza el consumo de energía, los movimientos de materiales y los costos. Emplear fuentes de energías renovables y menos contaminantes, constituyen también a la práctica sostenible. Por ejemplo; la reducción de algunas etapas del proceso de fabricación, la reducción de sus residuos, el incrementar el reciclaje, además de la utilización de menos consumibles o bien, buscar que estos sean más limpios.

En el número cuatro, esta, la optimización de los sistemas de distribución. Embalaje menor, más limpio y reutilizable. Modos de transporte energéticamente más eficientes, optimizando el espacio. Logística energéticamente más eficiente.

En el número cinco, busca la reducción del impacto ambiental durante su uso. Es importante, disminuir las emisiones de CO2, NOx y SOx, para evitar el efecto invernadero. Para lograrlo, se buscan componentes 
y recursos más eficientes que incluyan, asegurar un bajo consumo energético, buscar fuentes de energías limpias como la hidráulica, gas natural, solar, eólica. También, las reducciones de consumibles, así como consumibles limpios, incluyendo la reducción del consumo de agua, lubricantes, filtros. Esto, es muy necesario, se tome en cuenta durante el proceso del producto.

El número seis se centra en la Optimización de la vida del producto, misma que busca: alta fiabilidad y durabilidad, facilidad de mantenimiento y reparación, estructura de producto modular y adaptable, además de conseguir un diseño clásico; esto consiste en aprovechar algunas partes o componentes antes de que terminen su vida útil. Es por ello que deberá, considerar una fuerte relación, entre el producto y el usuario.

El número siete busca, Optimización del fin de vida del sistema, favorecer la reutilización del producto completo, o lo que se entiende como su re-fabricación o reacondicionamiento, esto permite favorecer el reciclaje, además de una incineración segura.

Finalmente, el número ocho, que se centra en el Desarrollo de nuevos conceptos, además, desmaterialización, el uso compartido del producto, la búsqueda de integración de funciones, la optimización funcional del producto.

\section{Sostenibilidad y sustentabilidad}

Las palabras "sostenible" y "sustentable" suelen emplearse como sinónimos al momento de hablar sobre temas ecológicos y ambientales. Ambos términos, se refieren a algo que logra sostenerse o sustentarse por sí mismo. Aplicados al campo de la economía, la ecología o el desarrollo y responsabilidad social, el desarrollo sostenible y el desarrollo sustentable son sinónimos. Sin embargo, precisemos un poco esto.

La "sustentabilidad" es un término ligado a la acción del hombre en relación con su entorno; es algo que podrá sostenerse o sustentarse por sí mismo. Dentro de la disciplina ecológica, se refiere a los sistemas biológicos que pueden conservar la diversidad y productividad a lo largo del tiempo. Se define sustentabilidad como "La capacidad de satisfacer necesidades de la generación humana actual sin que esto suponga la anulación de que las generaciones futuras también puedan satisfacer las necesidades propias" en el informe de Brundtland 1987 (Bermejo. 2014).

La sustentabilidad está ligada al concepto de desarrollo humano, el cual busca ser sostenible. Se enfoca principalmente en la preservación de los recursos naturales, para que futuras generaciones puedan disfrutar de estos. Este tema, apareció por primera vez en un debate político internacional en 1980, introducido por el grupo de trabajo: Estrategia para la conservación del planeta dependiente del Programa de las Naciones Unidas para el Medio Ambiente (UNEP). Brundtland, mencionado anteriormente es quien presentó este modelo de desarrollo universalmente en 1987, en su informe: Nuestro Futuro Común.

El término, sostenibilidad se define como "el uso equilibrado del capital natural, social y económico para lograr el bienestar continuado del planeta y de las generaciones futuras" (Mikesell, 2002). Es decir, será todo aquello que pueda sostenerse por sí mismo, basado en las condiciones económicas, política, medio ambiente y socioculturales. Esto hace que incorpore una visión humana, para que al satisfacer sus necesidades no descuide el impacto al entorno natural en el que habita: 
- En lo social, buscará contribuir, para que las personas se organicen para satisfacer sus necesidades básicas como alimentación, vivienda, seguridad, salud, educación, trabajo, recreación, por mencionar algunas; y esto será, a través de facilitar información, objetos y espacios; de tal modo que la interacción pueda llegar a ser: armónica, justa, equitativa, incluyente promoviendo el desarrollo integral de todos los miembros de su sociedad.

- En el aspecto ambiental, deberá orientar la manera en que la sociedad interactúa con el medio ambiente: cómo dispone de los bienes que la naturaleza proporciona; cómo cuida y hace uso de los recursos: agua, aire, suelo; y todo lo que de allí proviene para el bienestar de todos. Asimismo, deberá informar, cómo se obtienen los recursos del medio ambiente, cómo se mantiene el equilibrio en los procesos productivos, los materiales de insumo y las energías que emplean para ello

- Con respecto al factor económico, buscará contribuir con la generación de riqueza y de atribución de valor a las cosas dentro del intercambio de recursos en la sociedad. Además, de colaborar en la manera en que esa riqueza se distribuye y busque beneficiar a todos los sectores de la sociedad de manera equitativa, para una mejorar calidad de vida. También, deberá tener un impacto relevante en la generación de productos para el consumo, en sus estrategias de promoción, pero también, en el desarrollo de una cultura de consumo racional.

- Finalmente, en el factor político, buscará colaborará con el desarrollo de estructuras más democráticas, participando en estrategias de formación y desarrollo democrático, que permitan la inclusión de todos los miembros de la comunidad. Contribuirá con la orientación para transparentar el ejercicio de los poderes económicos y políticos. Así como, el desarrollo de leyes y reglamentos en todos los niveles institucionales que protejan a la sociedad y al ambiente.

\section{Conclusiones}

El trabajo de los diseñadores y de las empresas está íntimamente ligado, aquí pudimos apreciar en un repaso muy rápido, de cuáles son las áreas en las que el diseñador participa, la responsabilidad que se transfiere en todos y cada uno de sus procesos, el trabajo multidisciplinario, así como la relevancia de la comunicación.

La relación Empresa-Diseño, va más allá de generar una imagen, una identidad o establecer un posicionamiento en el mercado. Implica una responsabilidad social, y esta, es tarea de todos, es reconocer y aceptar los compromisos que se tienen con el entorno y la sociedad. La cual deberá presentar una actitud madura, consciente y sensible a los problemas que enfrenta, con actitud proactiva, para adoptar hábitos, estrategias y procesos que ayuden a minimizar los impactos negativos que se puede generar. Ser responsables es estar consiente y empático a lo que pasa en su comunidad interna, externa local y a nivel

global. Cada una de las profesiones, debe estar consciente de su responsabilidad social y el diseño no está exento de esto: 
"La responsabilidad del diseñador (...) su buen juicio social y moral tiene que entrar en juego mucho antes de que empiece a diseñar, porque tiene que juzgar, apriorísticamente, además si los productos que se le pide que diseñe o rediseñe merecen su atención o no. En otras palabras, si su diseño está a favor o en contra de un bien social" (Papanek, 1971, p. 57).

Todos estos conceptos trabajan juntos y van relacionados durante su implementación al lograr que el producto, proceso, o servicio, sea capaz de sostenerse o sustentarse por sí solo y a su vez nos referimos a un proceso que puede mantenerse sin afectar a la generación actual o futura. Este tipo de visión busca, cubrir las necesidades actuales, pero siempre tomando en cuenta a las generaciones futuras.

La sostenibilidad es un objetivo que está en constante cambio debido a la evolución de los materiales y procesos. Actualmente, diseñadores y organizaciones han creado una gran gama de recursos dentro de la red de los cuales se puede sacar gran provecho. Dentro de estos sitios web se puede encontrar información acerca de imprentas responsables con el medio ambiente, nuevos productos papeleros e información sobre las tendencias de diseño sostenibles.

Los diseñadores, como hemos venido revisando y aplicando prácticas sostenibles en los diferentes niveles en los que se participa; incluso, aquellos profesionales que ya tienen tiempo en el ámbito del diseño, debe de buscar un equilibrio dentro de las necesidades básicas y económicas contemplando así en una totalidad las implicaciones sociales y económicas de los materiales utilizados, el diseño y los procesos de producción, como con las necesidades mimas del planeta independientemente si se trata de una empresa pequeña mediana o grande, nacional o transnacional.

Finalmente cabe mencionar que, la importancia de la responsabilidad social radica como menciona Remolina (2000), citado por Barrera (2004), "el ser humano por su misma índole es social y debe conformar el tejido de sus relaciones en forma de convivencia justa y equitativa, de modo que viva y se desarrolle en un ambiente de convivencia y de calidad de vida verdaderamente humana".

Entender el rol y la responsabilidad, son términos que debieran estar insertos en la conducta cotidiana, en el ser y el hacer del diseño, que va dando como respuesta, un impacto en la identidad cultural de la sociedad. Buscar un cambio con la intención de mejorar, es una tarea que se construye con el trabajo diario, si bien es importante saber a quién y para que se diseña, tomando en cuenta las consideraciones anteriormente mencionadas; requerirá de un trabajo detallado, organizado, bajo el apoyo de diversos procesos y métodos, mismos que permitan la integración de diversos actores, con la intención de abarcar problemáticas cada vez más complejas.

\section{Referencias}

Barrera, G. y Remolina (2004). Diseño con Responsabilidad Social. España: Facultad de Arquitectura Pontificia Universidad Javeriana.

Bermejo, R. (2014). Del Desarrollo Sostenible Según Brundtland a la Sostenibilidad como Biomimesis. Bilbao: Editorial Hegoa.

Brezet, H. y Hemel, V. (1997). C Ecodesign: a promising approach to sustainable production and consumption. París: United Nations Environment Programme Industry and Environment UNEP IE. 
Capriotti, P. (2013). Planificación Estratégica de la Imagen Corporativa. Málaga, España: Editorial Instituto de Investigación en Relaciones Publicas.

Chaves, N. (2003). La imagen corporativa, Teoría y práctica de la identificación institucional. México: Editorial Gustavo Gill.

Kolter, P. y Amstrong, G. (2003). Principios de Marketing. México: Editorial. Pearson Prentice Hall.

Mikesell, R. (1995). The theory of Common Markets as applied to Regional Arrangements Among Developing Countries, en Harvard and Hague. New York: Editorial. St. Martin's Press.

Papaneck, V. (1972). Diseño para un mundo real, Ecología Humana y cambio social. New York: Editorial. POL. LEN.

Press, M. y Cooper, R. (2009) Diseño como Experiencia. El papel del diseño y los diseñadores en el siglo XXI. Barcelona: Editorial. Gustavo Gill SL.

Urrutia, A. A. (2000). Comunicación en la empresa La importancia de la información interna en la empresa. Revista Latina de comunicación social, 3(27), 0. 